\title{
Histopathologic correlation with diffusion tensor imaging after chronic hypoxia in the immature ferret
}

\author{
Joshua D. Tao' ${ }^{1}$ Alan R. Barnette', Jennifer L. Griffith², Jeffrey J. Neil ${ }^{3}$ and Terrie E. Inder ${ }^{1}$
}

INTRODUCTION: Chronic hypoxia in rodents induces white matter (WM) injury similar to that in human preterm infants. We used diffusion tensor imaging (DTI) and immunohistochemistry to study the impact of hypoxia in the immature ferret at two developmental time points relevant to the preterm and term brain.

RESULTS: On ex vivo imaging, the apparent diffusion coefficient (ADC) was decreased throughout the WM after 10 days of hypoxia (hypoxia from postnatal day 10 (P10) to P20 and killed at P20 (early hypoxia P20)), corresponding to increased astrocytosis and decreased myelination. Diffusion values normalized after 10 days of normoxia (hypoxia from P10 to P20 and killed at P30 (early hypoxia P30)), but immunohistochemistry revealed significant astrocytosis and hypomyelination. In contrast, ADC and anisotropy were increased after 10 days of hypoxia at a later developmental time point (hypoxia from P20 to P30 and killed at P30 (late hypoxia P30)), with less astrocytosis and more prominent myelination.

DISCUSSION: The patterns of alteration in imaging and histology varied in relation to the developmental time at which hypoxia occurred. Normalization of diffusion measures did not correspond to the normalization of underlying histopathology.

METHODS: Ferrets were subjected to 10\% hypoxia and divided into three groups: early hypoxia P20, early hypoxia P30, and late hypoxia P30.

B ecause of the increasing number of very-low-birth-weight infants surviving with motor, cognitive, and behavioral disabilities, a suitable animal model of preterm brain injury would be of great value. Prior work in rodents has shown that chronic hypoxia produces ventriculomegaly, reduced cortical volumes, and reduced neuronal size $(1,2)$. Histological studies have demonstrated that hypoxia has an adverse impact on oligodendrocytes and axonal connections in the developing rodent brain $(3,4)$. In this study, we use the immature ferret to expand on these rodent models and characterize the impact of hypoxia using diffusion tensor imaging (DTI) on fixed tissue followed by histopathology.

Unlike the rodent, the ferret is born lissencephalic and undergoes cortical folding during the first few postnatal weeks. Myelination, gyral folding, and forebrain development all occur postnatally, which differentiates the ferret from other models and allows for a unique period of study or intervention. Additionally, microstructural development occurs in a fashion similar to that in the human brain, making the ferret a potentially valuable model for brain injury in preterm human infants $(5,6)$. The ferret is also amenable to study in a laboratory setting. Its relatively small size allows it to fit easily into standard magnetic resonance imaging (MRI) scanners, and it is relatively inexpensive compared with the larger, more complex animals requiring intensive care support.

This work characterizes the pattern of brain injury and alterations in brain development in the immature ferret resulting from chronic hypoxia. We sought to use MRI with DTI to determine the effect of hypoxia at two key developmental time points in ferret brain maturation and to investigate the histopathological correlations of diffusion parameters. Postnatal day 10 (P10) in the ferret is equivalent to a human infant at 25-wk gestation, whereas P20 is equivalent to human term (5). We hypothesized that chronic, sublethal hypoxia would produce different patterns of white matter (WM) injury based on the timing of the hypoxic insult relative to the developmental stage of the brain.

\section{RESULTS}

\section{Overall Growth}

Body and brain weights of control and experimental animals were obtained at the time of killing (Table 1). Ferrets in the early hypoxia P20 group trended toward lower body and brain weights when compared with age-matched controls. In the early hypoxia P30 group, experimental animals displayed a trend toward reduced body weight compared with P30 controls, but a similar trend was not seen in brain weight. For the late hypoxia P30 group, there was a decrease $(P<0.02)$ in body weight compared with controls, with a trend toward decreased brain weight.

\section{MRI}

As seen in Figure 1, cortical folding is more complex, with deeper sulci and an increased number of gyri, in more mature brains. T2 hyperintensity is noted throughout the WM at the 
Table 1. Mean body and brain weights of control and experimental animals

\begin{tabular}{|c|c|c|c|c|c|}
\hline & \multicolumn{2}{|c|}{ P20 animals } & \multicolumn{3}{|c|}{ P30 animals } \\
\hline & Control & Early hypoxia & Control & Early hypoxia & Late hypoxia \\
\hline $\begin{array}{l}\text { Body weight } \pm \text { SEM }(\mathrm{g}) \\
(P \text { value })\end{array}$ & $\begin{array}{c}81.0 \pm 7.14 \\
(-)\end{array}$ & $67.6 \pm 1.94$ (NS) & $\begin{array}{c}141.0 \pm 6.35 \\
(-)\end{array}$ & $\begin{array}{l}120.0 \pm 11.0 \\
(0.14)\end{array}$ & $\begin{array}{c}102.7 \pm 5.23 \\
(<0.02)\end{array}$ \\
\hline $\begin{array}{l}\text { Brain weight } \pm \text { SEM ( } \mathrm{g}) \\
(P \text { value })\end{array}$ & $\begin{array}{c}2.79 \pm 0.72 \\
(-)\end{array}$ & $2.75 \pm 0.05$ (NS) & $\begin{array}{c}4.59 \pm 0.11 \\
(-)\end{array}$ & $\begin{array}{l}4.69 \pm 0.20 \\
\text { (NS) }\end{array}$ & $\begin{array}{c}4.32 \pm 0.08 \\
\text { (NS) }\end{array}$ \\
\hline
\end{tabular}

$P$ values represent comparisons with age-matched controls.

NS, not significant; P20, postnatal day 20; P30, postnatal day 30.

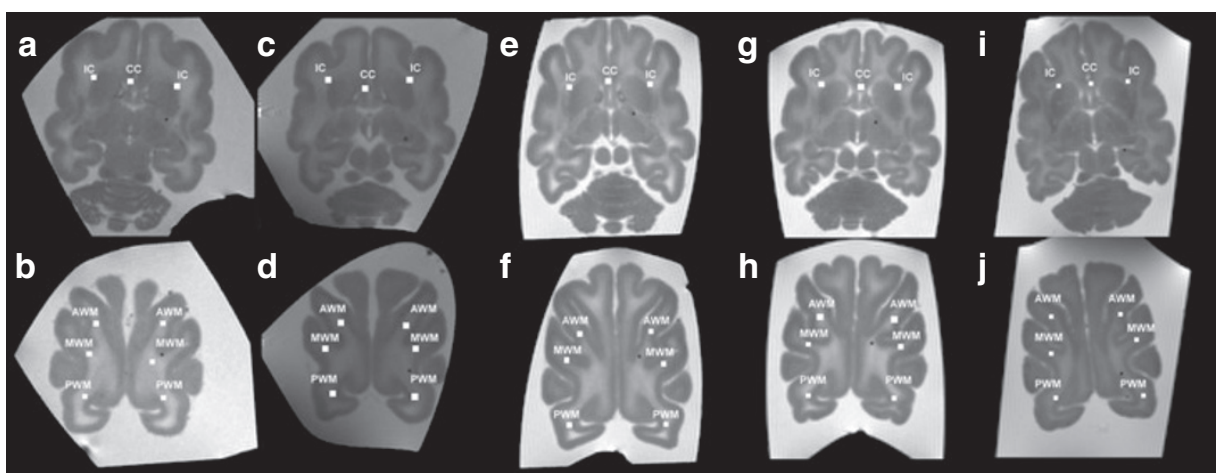

Figure 1. T2-weighted images of control and hypoxic ferret brains. Axial slices at the level of the midcaudate (top) and centrum semiovale (bottom) with representative regions of interest are shown in select WM tracts. (a, b) P20 control; (c, d) early hypoxia P20; (e, f) P30 control; (g, h) early hypoxia P30; and (i, j) late hypoxia P30. AWM, anterior white matter; CC, corpus callosum; IC, internal capsule; MWM, middle white matter; P20, postnatal day 20; P30, postnatal day 30; PWM, posterior white matter; WM, white matter.

level of the caudate and the centrum semiovale at P20, with less hyperintensity at P30. Areas of hypointensity, caused by myelination, are increasingly evident at P30 throughout the cerebral WM. There was no focal WM pathology or other types of injury noted on T2-weighted images of hypoxic animals.

In relation to hypoxic exposure, ferrets in the early hypoxia P20 group had reduced apparent diffusion coefficient (ADC; $P<0.01)$ throughout the WM in the centrum semiovale, internal capsule, and corpus callosum compared with agematched controls. No changes in relative anisotropy (RA) were noted. Both axial and radial diffusivity were decreased in all WM areas $(P \leq 0.02)$. The results of ADC and RA are displayed in Figure 2.

Immature ferrets subjected to hypoxia from P10 to P20 that were then maintained in a normoxic environment for 10 days (early hypoxia P30) were similar to their age-matched controls. There were no differences in ADC, RA, axial, or radial diffusivity in any region. Specifically, differences seen in the early hypoxia P20 group did not persist after 10 days of normoxia.

Ferrets subjected to hypoxia at a later age (late hypoxia P30) showed a different pattern of diffusion abnormalities. ADC values were higher compared with P30 controls in all areas of the centrum semiovale $(P<0.01)$ but not in the corpus callosum or internal capsule. RA was higher in the posterior centrum semiovale and internal capsule $(P \leq 0.02)$ but not in other areas. The higher RA values were the result of a greater percentage change in axial vs. radial diffusivity in the posterior centrum semiovale
$(+33 \%$ vs. $+16 \%)$ and internal capsule ( $+18 \%$ vs. $-8 \%)$. Absolute differences and percentage change between control and experimental groups are shown in Table 2.

\section{Immunohistochemistry}

GFAP. Representative images are shown in Figures 3 and 4. The brains of the ferrets in the early hypoxia P20 group $(n=3)$ showed an increased density of astrocytes compared with controls $(n=2), 129 \pm 18$ vs. $31 \pm 1$ cells $/ \mathrm{mm}^{2}(P<0.02$, Figure 5$)$. For the early hypoxia P30 group $(n=9)$, there was increased glial fibrillary acidic protein (GFAP) staining density compared with controls $\left(n=3,208 \pm 21\right.$ vs. $121 \pm 31$ cells $/ \mathrm{mm}^{2}$, $P<0.05)$. For late hypoxia P30 $(n=4)$, GFAP counts demonstrated a trend toward less astrocytes compared with controls (76 \pm 7 vs. $121 \pm 31$ cells $\left./ \mathrm{mm}^{2}, P=0.28\right)$ and were significantly less than in the early hypoxia P30 group $(P<0.01)$.

$M B P$. Representative images are shown in Figures 6 and 7. The early hypoxia P20 group $(n=2)$ had less myelin basic protein (MBP) staining in the periventricular WM tracts than the age-matched controls $(n=2)$. For P30 comparisons, early hypoxia P30 $(n=5)$ demonstrated less subcortical myelination and more areas of discontinuous myelin staining compared with controls $(n=3)$. In contrast, late hypoxia P30 brains $(n=4)$ showed thicker, more continuous WM tracts and an increase in subcortical myelination compared with controls. Semiquantitative evaluation in the P30 animals showed a lower total WM score in early hypoxia $\mathrm{P} 30$ compared with late hypoxia P30 ( $2.1 \pm 0.38$ vs. $7.1 \pm 0.64, P<0.01)$. 


\section{Articles | Chronic hypoxia in the immature ferret}
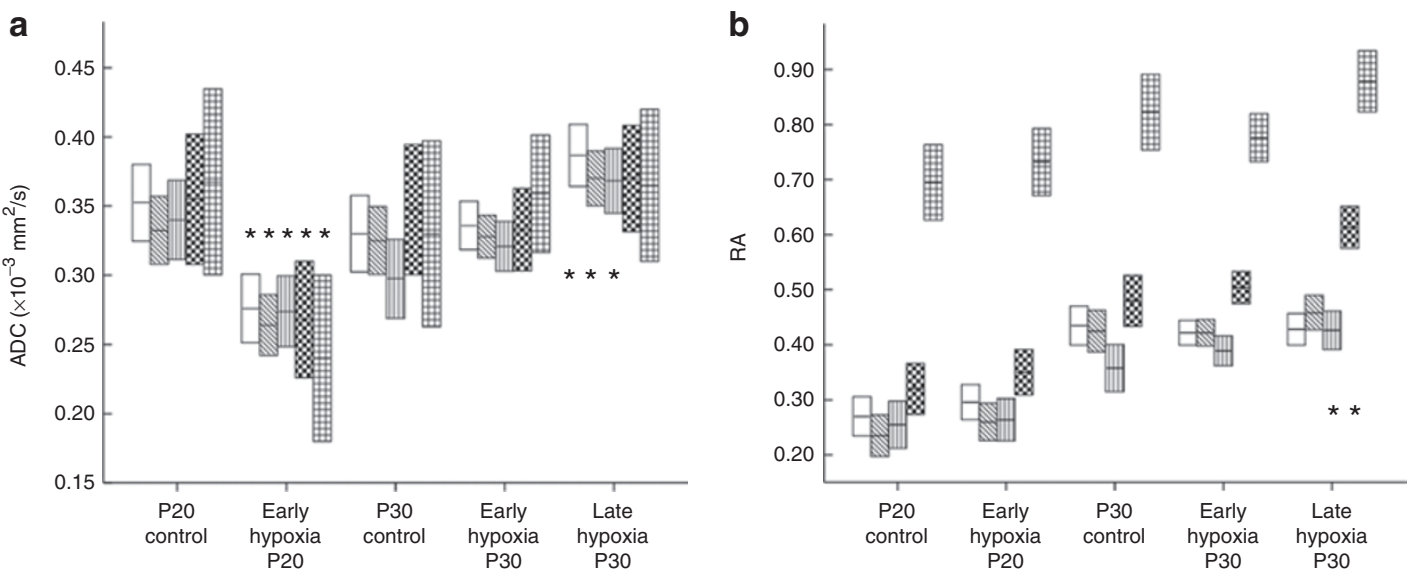

Figure 2. White matter (WM) diffusion measures are influenced by the timing of hypoxia. (a) Changes in WM ADC in control and experimental groups. (b) Changes in WM RA in control and experimental groups. Bars and lines represent the mean and 95\% confidence interval for each region. Open, anterior WM; cross-hatched, middle WM; vertical stripes, posterior WM; checkerboard, internal capsule; open grid, corpus callosum. ${ }^{*} P \leq 0.05$ compared with age-matched controls. ADC, apparent diffusion coefficient; P20, postnatal day 20; P30, postnatal day 30; RA, relative anisotropy.

Table 2. Absolute differences in mean diffusion measures between control and hypoxic brains (percentage change)

\begin{tabular}{|c|c|c|c|}
\hline & $\begin{array}{l}\text { P20 control- } \\
\text { early hypoxia P20 }\end{array}$ & $\begin{array}{l}\text { P30 control- } \\
\text { early hypoxia P30 }\end{array}$ & $\begin{array}{l}\text { P30 control-late } \\
\text { hypoxia P30 }\end{array}$ \\
\hline \multicolumn{4}{|c|}{ Apparent diffusion coefficient $\left(10^{-3} \mathrm{~mm}^{2} / \mathrm{s}\right)$} \\
\hline AWM & $0.0765(-22)^{* *}$ & $-0.0060(+2)$ & $-0.0567(+17)^{*}$ \\
\hline MWM & $0.0685(-21)^{* *}$ & $-0.0030(+1)$ & $-0.0450(+14)^{*}$ \\
\hline PWM & $0.0660(-19)^{*}$ & $-0.0235(+8)$ & $-0.0708(+24)^{* *}$ \\
\hline IC & $0.0870(-25)^{*}$ & $0.0145(-4)$ & $-0.0225(+6)$ \\
\hline $\mathrm{CC}$ & $0.1275(-35)^{*}$ & $-0.0290(+9)$ & $-0.0350(+11)$ \\
\hline \multicolumn{4}{|c|}{ Relative anisotropy } \\
\hline AWM & $-0.0260(+10)$ & $0.0130(-3)$ & $0.0067(-2)$ \\
\hline MWM & $-0.0250(+11)$ & $0.0030(-1)$ & $-0.0333(+8)$ \\
\hline PWM & $-0.0090(+4)$ & $-0.0315(+9)$ & $-0.0692(+19)^{*}$ \\
\hline IC & $-0.0300(+9)$ & $-0.0240(+5)$ & $-0.1333(+28)^{* *}$ \\
\hline CC & $-0.0370(+5)$ & $0.0465(-6)$ & $-0.0558(+7)$ \\
\hline \multicolumn{4}{|c|}{ Axial diffusivity $\left(10^{-3} \mathrm{~mm}^{2} / \mathrm{s}\right)$} \\
\hline AWM & $0.0830(-18)^{*}$ & $-0.0065(+1)$ & $-0.0742(+15)^{*}$ \\
\hline MWM & $0.0700(-17)^{*}$ & $-0.0005(0)$ & $-0.0842(+17)^{*}$ \\
\hline PWM & $0.0755(-17)^{*}$ & $-0.0460(+11)$ & $-0.1433(+33)^{* *}$ \\
\hline IC & $0.1185(-23)^{*}$ & $0.0150(-3)$ & $-0.1017(+18)^{*}$ \\
\hline $\mathrm{CC}$ & $0.2380(-33)^{*}$ & $-0.0300(+4)$ & $-0.1000(+14)$ \\
\hline \multicolumn{4}{|c|}{ Radial diffusivity $\left(10^{-3} \mathrm{~mm}^{2} / \mathrm{s}\right)$} \\
\hline AWM & $0.0695(-23)^{* *}$ & $-0.0035(+1)$ & $-0.0458(+19)^{*}$ \\
\hline MWM & $0.0655(-23)^{* *}$ & $-0.0015(+1)$ & $-0.0242(+10)^{*}$ \\
\hline PWM & $0.0590(-21)^{* *}$ & $-0.0145(+6)$ & $-0.0358(+16)^{*}$ \\
\hline IC & $0.0710(-26)^{*}$ & $0.0105(-5)$ & $0.0175(-8)$ \\
\hline $\mathrm{CC}$ & $0.0740(-39)^{*}$ & $-0.0260(+19)$ & $0.0050(-4)$ \\
\hline
\end{tabular}

Numbers in parentheses are the percentage change.

AWM, anterior white matter; CC, corpus callosum; IC, internal capsule; MWM, middle white matter; PWM, posterior white matter; P20, postnatal day 20; P30, postnatal day 30 . ${ }^{*} P \leq 0.05 ;{ }^{* *} P \leq 0.001$
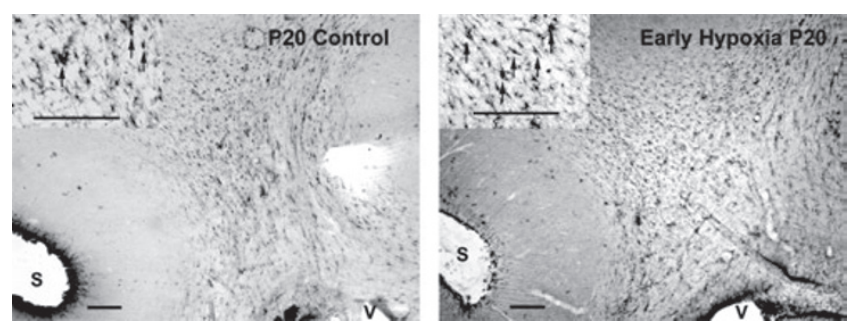

Figure 3. Glial fibrillary acidic protein staining at postnatal day 20 (P20) is increased after hypoxia. Low- and high-power views show an increased density of astrocytes (black arrows) in the early hypoxia P20 group. S, sulcus; V, ventricle. Bar $=250 \mu \mathrm{m}$.

\section{Comparison of Diffusion and Histology Measures}

When comparing absolute values, we found no correlation between diffusion and histology measures. However, when using groupwise comparisons, ADC was lower in brains with GFAP counts above the gestational control levels compared with brains presenting GFAP counts below control levels in the anterior $(0.34 \pm 0.01$ vs. $0.39 \pm 0.01, P=0.02)$, middle ( $0.30 \pm 0.01$ vs. $0.38 \pm 0.02, P<0.01)$, and posterior $(0.30 \pm$ 0.01 vs. $0.36 \pm 0.01, P<0.03) \mathrm{WM}$, as well as in the internal capsule $(0.30 \pm 0.02$ vs. $0.38 \pm 0.05, P<0.05)$. There was no difference in ADC when comparing brains with hypomyelination (total WM $<4$ ) and those with higher WM scores $(>4)$. RA was lower in brains with GFAP counts above control levels compared with brains presenting GFAP counts below control levels in the internal capsule $(0.47 \pm 0.03$ vs. $0.64 \pm 0.01, P<0.05)$ and corpus callosum $(0.77 \pm 0.02$ vs. $0.90 \pm 0.06, P=0.05)$. RA was lower in hypomyelinated brains (total WM score $<4$ ) compared with brains with higher WM scores $(>4)$ in the middle $\mathrm{WM}(0.40 \pm 0.03$ vs. $0.47 \pm 0.01, P=0.05)$ and corpus callosum (0.75 \pm 0.04 vs. $0.88 \pm 0.02, P<0.02)$.

\section{DISCUSSION}

This study characterizes the pattern of alterations in diffusion MRI measures and histopathological observations at two 

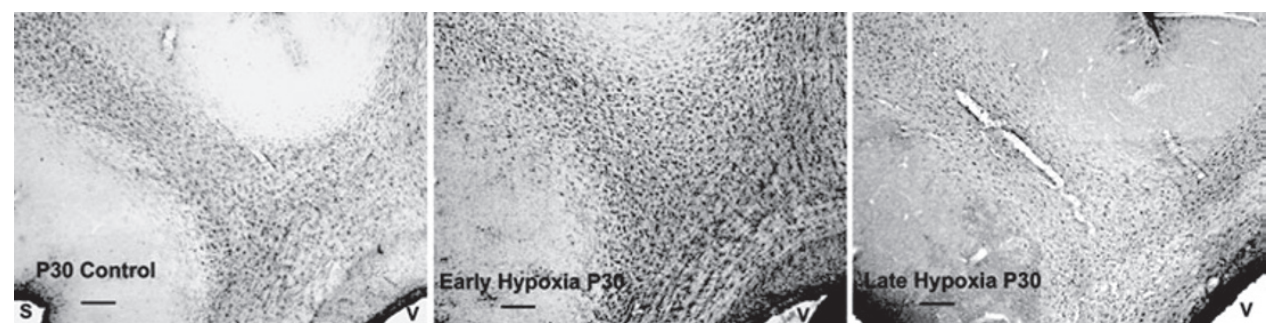

Figure 4. Glial fibrillary acidic protein staining at postnatal day 30 (P30) varies based on the timing of hypoxia. Astrocyte staining is present in coronal slices of control animals, increased in early hypoxia P30, and decreased in late hypoxia P30. S, sulcus; V, ventricle. Bar $=250 \mu \mathrm{m}$.

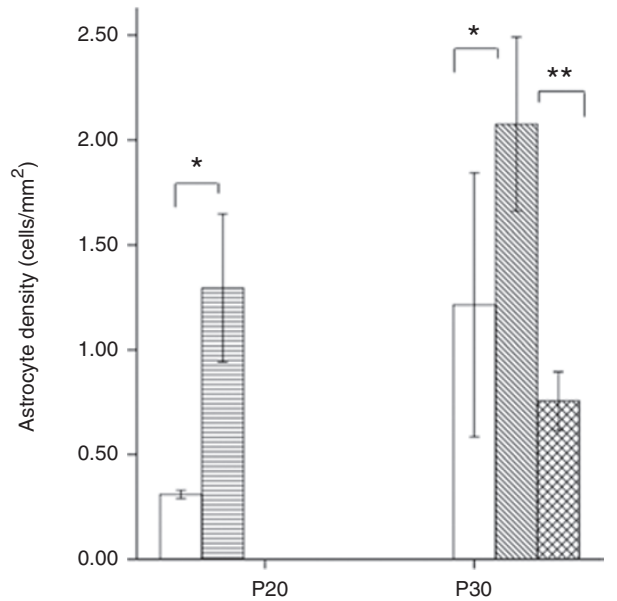

Figure 5. Astrocyte density in control vs. hypoxic brains. Open, control; horizontal stripes, early hypoxia P20; cross-hatched, early hypoxia P30; diagonal grid, late hypoxia P30. Bars and lines represent means \pm SEM. ${ }^{*} P<0.05 ;{ }^{* *} P<0.01$. P20, postnatal day 20; P30, postnatal day 30 .
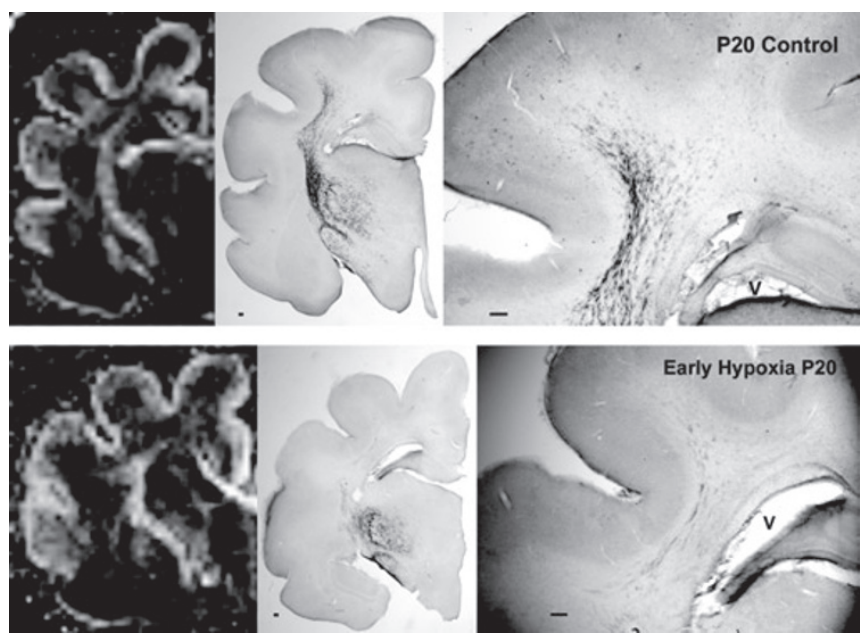

Figure 6. Comparison of anisotropy parametric maps and myelin basic protein (MBP) staining at P20. Relative anisotropy (RA) parametric maps (left) compared with MBP staining at low (middle) and high (right) power on coronal slices in control vs. hypoxic brains. Higher-intensity areas on parametric maps indicate higher RA. P20, postnatal day 20; V, ventricle. Bar $=250 \mu \mathrm{m}$.

relevant developmental time points in an immature ferret model of chronic hypoxia. Hypoxia at preterm equivalent age (P10 to P20) was associated with a reduction in WM diffusivity, an increase in astrocytes, and a decrease in myelination.
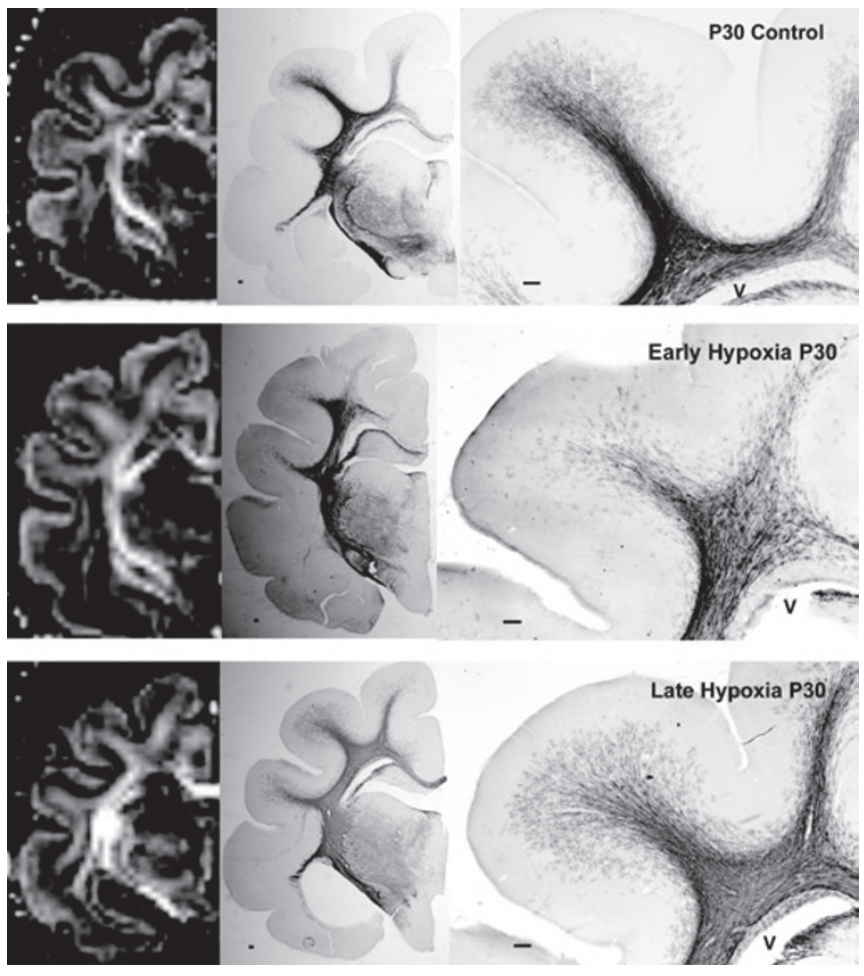

Figure 7. Comparison of anisotropy parametric maps and myelin basic protein (MBP) staining at P30. Relative anisotropy (RA) parametric maps (left) compared with MBP staining at low (middle) and high (right) power on coronal slices in control vs. hypoxic brains. Higher-intensity areas on parametric maps indicate higher RA. P30, postnatal day 30; V, ventricle. $\mathrm{Bar}=250 \mu \mathrm{m}$

Ferrets demonstrated normalization of these alterations in diffusion measures after a period of normoxia. However, this normalization was not reflected by the normalization of the underlying histopathology. Late hypoxia at term equivalent age (P20 to P30) resulted in a different injury pattern, with an increase in diffusivity and RA, a decrease in astrocytosis, and an increase in myelination.

Normal brain development in the ferret has been well described $(7,8)$. The most rapid phase of gyrification and an increase in cortical volume occur between P14 and P28. Thereafter, myelination predominates and continues into the fifth and sixth weeks of life $(5,6)$. Control ferrets in this study underwent changes in diffusion indices similar to those described for human preterm infants $(9,10)$. Specifically, RA increased, whereas ADC and radial diffusivity decreased in 


\section{Articles | Chronic hypoxia in the immature ferret}

WM over time from P20 to P30. Histologically, astrocytes were visible at P20 and remained prominent in P30 controls. Myelin staining was less prominent in the early stage, but was clearly present by $\mathrm{P} 30$.

In this study, it is important to bear in mind that imaging was obtained on fixed tissue. Specifically, the reduction in ADC detected early in rodent models of injury (11) is no longer detectable after fixation because ADC values for normal and injured tissues are equal (12). Thus, the changes in diffusion reflect chronic changes in tissue microstructure (which survive fixation) rather than a response to acute or subacute injury (which leads to a reduction in ADC that is lost after fixation).

Anisotropy values, on the other hand, are preserved after fixation (13) because fixation is associated with proportional reductions in axial and radial diffusivities. As a result, in both live and fixed brain, reductions in axial diffusivity are associated with axonal damage $(14,15)$, whereas increases in radial diffusivity are associated with damage to myelin $(14,16)$.

The early hypoxia P20 group was undertaken to resemble preterm brain injury by killing the ferret at term equivalent age. This model was associated with lower ADC values throughout the WM with no change in RA. Lower ADC values suggest greater cell density because the diffusion of water molecules is limited by an increased number of cell bodies, membranes, and processes. This was demonstrated histologically because an increased density of astrocytes was present in WM. However, decreased MBP staining was noted in this group as well, suggesting that the number of mature oligodendrocytes was reduced or that the myelin-producing ability of these cells was impaired. Although this would be expected to be associated with lower RA values, there was no change in the RA in this group. The discrepancy between the RA and MBP findings might be explained by offsetting changes in microglia or axonal packing.

When this model was placed into normoxia for 10 days and killed at P30 (early hypoxia P30), a different pattern was observed. $\mathrm{ADC}$ and RA were similar to that of controls, indicating that the measures normalized during the 10 days of normoxia. Normalization of ADC has been noted in both animal models subjected to varying mechanisms of injury $(17,18)$ and human infants $(19,20)$. Although this acute reduction in ADC after injury is related to physiologic (not structural) changes, the subsequent pseudonormalization is likely due to structural changes associated with lower tissue cell density. In this study, normalization of diffusion parameters was associated with increased astrocytes and hypomyelination. It is likely that the increase in cell density caused by astrocytosis and the reduction in cell density caused by the loss of myelin offset each other sufficiently to be associated with normal diffusion parameters. This finding underscores the challenges of interpreting DTI data in isolation.

In the late hypoxia P30 group, ferrets exposed to hypoxia at term equivalent age (P20 to P30) demonstrated higher ADC in the centrum semiovale. This increase resembles that observed in the later phase of hypoxic-ischemic or inflammatory injury (18). However, the model described herein employed hypoxia alone. Recent studies have shown similar increases in $\mathrm{ADC}$ in premature infants at term with extensive WM signal abnormalities $(21,22)$. In this study, increased ADC likely represents a loss of cellular elements. Thus, the decrease in astrocytic density correlates with the findings on DTI. Whether the astrocytes are undergoing apoptosis or necrosis or simply not proliferating cannot be determined from this study. However, this response likely is developmentally regulated, given the findings of increased astrocytes in the early hypoxia P20 group.

Unlike the other groups in our study, RA in the late hypoxia P30 group was higher in the posterior centrum semiovale and internal capsule (but not in other areas) because of the relatively higher axial vs. radial diffusivity. These findings in the centrum semiovale-higher ADC and RA in association with higher axial diffusivity-suggest accelerated WM maturation. This is consistent with the "discordant maturation" described in human infants with intrauterine growth restriction (23) and suggests that this model is relevant to infants with intrauterine growth restriction. It is worth noting that the increase in anisotropy and corresponding increase in myelination do not necessarily indicate functional improvement. Although hypoxia selectively affects oligodendrocyte precursor cells (24), the effect of remyelination or increased myelination after hypoxia has been shown in a few studies $(25,26)$. Interestingly, chronic hypoxia depletes the oligodendrocyte-related axonal outgrowth inhibitors in rodents (4). This may underlie the increase in myelination and anisotropy seen in this study.

Other animal studies utilizing chronic hypoxia show varied results, such as reductions in WM axial diffusivity and anisotropy (27) or recovery of reduced glia and cortical volume after a period of normoxia (28). The current study is unique in that it compares WM DTI measures and histopathology in a novel model.

It is worth noting that the histopathologic findings presented here are a local rather than a global assessment of injury. DTI measurements, however, were measured throughout subcortical and deep WM tracts, providing an overall evaluation of brain microstructure. Regardless, the results of both methodologies reveal a significant and varied response to injury. Statistical correlation between individual immunohistochemistry and diffusion parameters was not found in this study, but groupwise comparisons demonstrated that decreased ADC and RA were associated with astrocytosis and that decreased RA was associated with hypomyelination.

This study has several limitations. In some histology groups, as few as two animals were studied, and results should be interpreted accordingly. Environmental and systemic carbon dioxide levels were not measured, and fluctuations in these levels could contribute to brain injury. Ferret jills were exposed to the same experimental conditions as their kits, and thus the hypoxia could have had a significant impact on the nutritional status of the nursing mother and her milk content. Although not measured here, cortisol and related stress-response hormones play a significant role in brain development (29) and could have affected our results. More detailed histologic evaluations could also provide a better understanding of neuropathology in this model, specifically the role of microglia and neurons in response to injury. Finally, this study involves the 
imaging and analysis of fixed, rather than live, tissue with the attendant caveats outlined above. However, for our comparisons between the models, it is important to note that all specimens were treated in the same way, and thus the changes would be similar across all groups.

In summary, this study documents the changes in DTI parameters and immunohistochemistry related to the timing of chronic hypoxia in the immature ferret. Serial in vivo imaging, alongside further histopathology and behavioral testing, could provide more insight into the impact of chronic hypoxia in this model. This understanding could allow the evaluation of a more chronic model of adverse brain development consistent with that documented in human preterm infants. An array of both simple and complex animal models will assist in the investigation of the pathophysiologic and neuroprotective approaches to the preterm brain.

\section{METHODS}

All procedures were reviewed and approved by the Washington University Animal Studies Committee and performed at Washington University.

\section{Subjects}

Male ferret kits and their nursing jill were obtained from a commercial vendor (Marshall Bioresources, North Rose, NY) and delivered to a dedicated animal facility at Washington University on P7 or later. The jill and ferret kits were exposed to $10 \%$ hypoxia by placing them in a custom-designed, clear plastic hypoxic chamber (Biospherix, Lacona, NY) with continuous monitoring of oxygen levels. The ambient oxygen level was reduced to $10 \pm 0.2 \%$ using a blended air/ $\mathrm{N}_{2}$ gas mixture and adjusted continuously with a compact gas oxygen controller (Proox model 360; Biospherix). The chamber was opened twice weekly for routine cleaning and food and water replacement.

Experimental animals were divided into three groups: (i) early hypoxia P20 $(n=5)$, in which kits were reared under hypoxic conditions from P10 to P20 and killed at P20; (ii) early hypoxia P30 $(n=10)$, in which kits were reared under hypoxic conditions from P10 to P20, returned to a normoxic environment (room air), and killed at P30; and (iii) late hypoxia P30 $(n=6)$, in which kits were reared under hypoxic conditions from P20 to P30 and killed at P30. Hypoxic groups were compared with age-matched controls reared under normoxic conditions until P20 $(n=4)$ and P30 $(n=4)$.

\section{Brain Fixation}

Ferrets were anesthetized by intraperitoneal injection of $100 \mathrm{mg} / \mathrm{kg}$ sodium pentobarbital. Intracardiac perfusion of $0.1 \mathrm{~mol} / \mathrm{l}$ heparinized phosphate-buffered saline (PBS) was performed to clear the vascular system, followed by perfusion with $4 \%$ paraformaldehyde. Brains were removed from the skull, placed in paraformaldehyde, and stored at $4^{\circ} \mathrm{C}$ until imaging was complete.

\section{Imaging and Analysis}

We obtained high-resolution ex vivo $\mathrm{MR}$ images of control and hypoxic brains using a 4.7-T magnet controlled by a Varian (Palo Alto, CA) INOVA console. Brains were placed in either 20- or 35-ml plastic syringes filled with paraformaldehyde during the scans and maintained at room temperature throughout the study. T2-weighted images (Figure 1) were acquired using the following parameters: $\mathrm{TR}=4.0-7.5 \mathrm{~s}, \mathrm{TE}=85(\mathrm{P} 20)$ or $93 \mathrm{~ms}(\mathrm{P} 30)$, voxel size $250(\mathrm{P} 20)$ or $300 \mu \mathrm{m}$ (P30), all isotropic.

Diffusion-weighted images were acquired using a 25-direction sampling scheme in which gradient strength was varied across each of the 25 gradient orientations. The values of $b$ ranged from 187 to
$11,300 \mathrm{~s} / \mathrm{mm}^{2}, \mathrm{TE}=72 \mathrm{~ms}, \mathrm{TR}=4.2-6.0 \mathrm{~s}$, voxel size $=250(\mathrm{P} 20)$ or $300(\mathrm{P} 30) \mu \mathrm{m}$ isotropic, diffusion gradient duration $=11.5 \mathrm{~ms}$, delay between diffusion gradients $=50 \mathrm{~ms}$, and diffusion gradient amplitude $=3-25 \mathrm{G} / \mathrm{cm}$. Raw diffusion data were phase corrected. RA, ADC, axial, and radial diffusivity parametric maps were calculated.

Using Analyze 9.0 (Rochester, MN) software, regions of interest (ROIs) were placed bilaterally by a single investigator (J.D.T.) in two horizontal slices of each brain (Figure 1). At the level of the caudate nuclei, ROIs were placed bilaterally in the internal capsule, and a single ROI was placed at the genu of the corpus callosum. Anterior, middle, and posterior WM regions were evaluated in the centrum semiovale. To minimize partial volume averaging, T2, ADC, and RA maps were compared side by side when placing ROIs.

\section{Immunohistochemistry}

After imaging, brains were stored at $4^{\circ} \mathrm{C}$ in $0.1 \mathrm{M}$ PBS. Only the left cerebral hemisphere was used for this analysis. Blocks were placed in agar overnight and serial coronal sections $(70 \mu \mathrm{m})$ were cut using a vibratome slicer. Five slices were chosen at consistent intervals throughout each brain, and the tissue was quenched in $0.3 \%$ hydrogen peroxide with methanol for $10 \mathrm{~min}$ followed by washing with PBS $(3 \times)$. Tissue was preblocked with $3 \%$ bovine serum albumin (BSA) in PBS with $0.8 \%$ Triton X-100 for $1 \mathrm{~h}$ at room temperature. Sections were incubated overnight with either rabbit anti-GFAP antibody (1:600 in 3\% BSA; Sigma, St Louis, MO) or rat anti-MBP antibodies ( $1: 100$ in 3\% BSA; Millipore, Billerica, MA) at room temperature. The following day, sections were incubated with either goat antirabbit secondary antibodies (1:200 in 3\% BSA; Sigma) or goat antirat secondary antibodies (1:200 in 3\% BSA; Sigma) for $60 \mathrm{~min}$, rinsed in PBS (3×), and incubated in an avidin-biotin complex for $60 \mathrm{~min}$. Development in Vector VIP solution (Vector Laboratories, Burlingame, CA) was then performed. Sections were rinsed in PBS $(2 \times)$, dehydrated, cleared with Citri Solv, and mounted in preparation for analysis.

\section{Astrocyte Assessment}

Control and experimental brains were assessed for astrocytes by a single, blinded investigator (J.D.T.). ROIs were placed in the periventricular WM on midcoronal slices. Density counts (number of astrocytes per square millimeter) were performed using a light microscope and Stereo Investigator 7.0 software (MBF Bioscience, Williston, VT) at $20 \times$ magnification. Cells with darkly stained cell bodies and two or more processes were identified as astrocytes.

\section{Myelin Assessment}

P30 brains were examined for the presence of MBP staining on light microscopy, and a semiquantitative analysis was performed using a point-counting technique utilized previously (30). Using the midcoronal slice, the internal capsule, corpus callosum, and subcortical WM were examined. Control brains were given a score of 2 in each of these areas, and the extent of myelination in experimental groups was assessed (0-3) based on this standard. Two researchers (J.D.T. and T.E.I.) performed the analysis independently and were blinded to the treatment groups. Interrater reliability was good $(\kappa=0.64)$, and the total WM score by each researcher was used to calculate the mean score.

\section{Statistics}

For MR images, bilateral ROIs were averaged, providing a single value for each region. For immunohistochemistry, GFAP cell density counts or WM scores were averaged within each group. For comparisons between diffusion and histology values, Pearson's correlation coefficient was used. GFAP density results were separated into three groups (less than the gestational control range, within the control range, and greater than the control range) and MBP results were separated into two groups (total WM score above or below 4) to perform groupwise comparisons with diffusion measures. A $t$ test or 
ANOVA was performed as indicated using SPSS 17.0 (SPSS, Chicago, IL). Significance level was set at $P<0.05$ using a two-tailed approach. Measurements described in the text are means \pm SEM unless otherwise indicated.

\section{ACKNOWLEDGMENTS}

We thank Nuri Farber and Shanna Zhang for their assistance with histopathology.

\section{STATEMENT OF FINANCIAL SUPPORT}

J.N.N. was supported by the Green Foundation.

\section{REFERENCES}

1. Ment LR, Schwartz M, Makes RW, Stewart WB. Association of chronic sublethal hypoxia with ventriculomegaly in the developing rat brain. Brain Res Dev Brain Res 1998;111:197-203.

2. Schwartz ML, Vaccarino F, Chacon M, Yan WL, Ment LR, Stewart WB. Chronic neonatal hypoxia leads to long term decreases in the volume and cell number of the rat cerebral cortex. Semin Perinatol 2004;28: 379-88.

3. Akundi RS, Rivkees SA. Hypoxia alters cell cycle regulatory protein expression and induces premature maturation of oligodendrocyte precursor cells. PLoS ONE 2009;4:e4739.

4. Weiss J, Takizawa B, McGee A, et al. Neonatal hypoxia suppresses oligodendrocyte Nogo-A and increases axonal sprouting in a rodent model for human prematurity. Exp Neurol 2004;189:141-9.

5. Barnette AR, Neil JJ, Kroenke CD, et al. Characterization of brain development in the ferret via MRI. Pediatr Res 2009;66:80-4.

6. Neal J, Takahashi M, Silva M, Tiao G, Walsh CA, Sheen VL. Insights into the gyrification of developing ferret brain by magnetic resonance imaging. J Anat 2007;210:66-77.

7. McSherry GM. Mapping of cortical histogenesis in the ferret. J Embryol Exp Morphol 1984;81:239-52.

8. McSherry GM, Smart IH. Cell production gradients in the developing ferret isocortex. J Anat 1986;144:1-14.

9. McKinstry RC, Mathur A, Miller JH, et al. Radial organization of developing preterm human cerebral cortex revealed by non-invasive water diffusion anisotropy MRI. Cereb Cortex 2002;12:1237-43.

10. Neil JJ, Shiran SI, McKinstry RC, et al. Normal brain in human newborns: apparent diffusion coefficient and diffusion anisotropy measured by using diffusion tensor MR imaging. Radiology 1998;209:57-66.

11. Derugin N, Wendland M, Muramatsu K, et al. Evolution of brain injury after transient middle cerebral artery occlusion in neonatal rats. Stroke 2000;31:1752-61.

12. Sun SW, Neil JJ, Liang HF, et al. Formalin fixation alters water diffusion coefficient magnitude but not anisotropy in infarcted brain. Magn Reson Med 2005;53:1447-51.

13. Sun SW, Neil JJ, Song SK. Relative indices of water diffusion anisotropy are equivalent in live and formalin-fixed mouse brains. Magn Reson Med 2003;50:743-8.
14. Song SK, Sun SW, Ju WK, Lin SJ, Cross AH, Neufeld AH. Diffusion tensor imaging detects and differentiates axon and myelin degeneration in mouse optic nerve after retinal ischemia. Neuroimage 2003;20:1714-22.

15. Beaulieu C, Does MD, Snyder RE, Allen PS. Changes in water diffusion due to Wallerian degeneration in peripheral nerve. Magn Reson Med 1996; 36:627-31.

16. Song SK, Sun SW, Ramsbottom MJ, Chang C, Russell J, Cross AH. Dysmyelination revealed through MRI as increased radial (but unchanged axial) diffusion of water. Neuroimage 2002;17:1429-36.

17. Rumpel H, Nedelcu J, Aguzzi A, Martin E. Late glial swelling after acute cerebral hypoxia-ischemia in the neonatal rat: a combined magnetic resonance and histochemical study. Pediatr Res 1997;42:54-9.

18. Lodygensky GA, West T, Stump M, Holtzman DM, Inder TE, Neil JJ. In vivo MRI analysis of an inflammatory injury in the developing brain. Brain Behav Immun 2010;24:759-67.

19. McKinstry RC, Miller JH, Snyder AZ, et al. A prospective, longitudinal diffusion tensor imaging study of brain injury in newborns. Neurology 2002;59:824-33.

20. Rutherford M, Counsell S, Allsop J, et al. Diffusion-weighted magnetic resonance imaging in term perinatal brain injury: a comparison with site of lesion and time from birth. Pediatrics 2004;114:1004-14.

21. Cheong JL, Thompson DK, Wang HX, et al. Abnormal white matter signal on MR imaging is related to abnormal tissue microstructure. AJNR Am J Neuroradiol 2009;30:623-8.

22. Counsell SJ, Shen Y, Boardman JP, et al. Axial and radial diffusivity in preterm infants who have diffuse white matter changes on magnetic resonance imaging at term-equivalent age. Pediatrics 2006;117:376-86.

23. Dubois J, Benders M, Borradori-Tolsa C, et al. Primary cortical folding in the human newborn: an early marker of later functional development. Brain 2008;131(Pt 8):2028-41.

24. Back SA, Han BH, Luo NL, et al. Selective vulnerability of late oligodendrocyte progenitors to hypoxia-ischemia. J Neurosci 2002;22:455-63.

25. Meng S, Qiao M, Scobie K, Tomanek B, Tuor UI. Evolution of magnetic resonance imaging changes associated with cerebral hypoxia-ischemia and a relatively selective white matter injury in neonatal rats. Pediatr Res 2006;59(4 Pt 1):554-9.

26. Sedowofia K, Giles D, Wade J, et al. Myelin expression is altered in the brains of neonatal rats reared in a fluctuating oxygen atmosphere. Neonatology 2008;94:113-22.

27. Chahboune H, Ment LR, Stewart WB, et al. Hypoxic injury during neonatal development in murine brain: correlation between in vivo DTI findings and behavioral assessment. Cereb Cortex 2009;19:2891-901.

28. Fagel DM, Ganat Y, Silbereis J, et al. Cortical neurogenesis enhanced by chronic perinatal hypoxia. Exp Neurol 2006;199:77-91.

29. Murphy BP, Inder TE, Huppi PS, et al. Impaired cerebral cortical gray matter growth after treatment with dexamethasone for neonatal chronic lung disease. Pediatrics 2001;107:217-21.

30. Loeliger M, Inder T, Cain S, et al. Cerebral outcomes in a preterm baboon model of early versus delayed nasal continuous positive airway pressure. Pediatrics 2006;118:1640-53. 